\title{
A QUESTION OF STARK
}

\author{
Brett A. TANGEDAL
}

One of the programs of Stark's conjectures is to find as many connections as possible between the values that Artin $L$-functions or their derivatives take (especially at $s=0$ ) and arithmetic information associated to algebraic number fields. The most refined of Stark's conjectures involves the values of first derivatives of $L$-functions at $s=0$. It was recognized early on that the conjecture should be extended to cover cases where the order of vanishing of the $L$-functions at $s=0$ is greater than one. In 1980, Stark posed a question along these lines that we will consider in detail here. In particular, we will study his question for relative quadratic extensions and prove that an affirmative answer to his question exists for all cases considered.

\section{Introduction.}

Our aim in this section is to state Stark's question and see how it is related to his refined conjecture. Throughout this paper $k$ will be a real quadratic number field and $K$ will denote an abelian extension of $k$. We consider $K$ and $k$ to be subfields of the complex numbers $\mathbb{C}$. Three different cases arise: i) Both infinite primes of $k$ split in $K$, i.e. $K$ is totally real. ii) One infinite prime of $k$ ramifies and the other splits. iii) Both infinite primes ramify. Stark's question involves case i). His refined conjecture deals with cases ii) and iii) (technically it deals with case i) as well but it doesn't tell us too much as we'll see below). We use the following notation for all three cases. $S$ will denote a finite set of primes in $k$ satisfying the following assumptions:

(a) It contains both infinite primes,

(b) it contains all finite primes that ramify in $K$,

(c) it contains a distinguished set of $r$ primes that split completely in $K$,

(d) the cardinality of $S,|S|$, satisfies $|S| \geq r+1$.

In cases ii) and iii) we will set $r=1$ in the following way: ii) $S=\left\{\infty_{k}, \bar{\infty}_{k}\right.$, $\left.\mathfrak{q}_{1}, \cdots, \mathfrak{q}_{t}\right\}$, where $\infty_{k}$ is the infinite prime corresponding to the identity embedding of $k$ in $\mathbb{R}$ and $\bar{\infty}_{k}$ is the conjugate prime. The $\mathfrak{q}_{i}$ are finite primes which include among them all of the ramified primes and we have $t \geq 0$. By assumption, exactly one of the infinite primes split and this will be our 
distinguished prime which we denote by p. iii) $S=\left\{\infty_{k}, \bar{\infty}_{k}, \mathfrak{q}_{1}, \cdots, \mathfrak{q}_{t}, \mathfrak{p}\right\}$, where since both infinite primes ramify by assumption, we add on as our one distinguished prime a finite prime $\mathfrak{p}$ that splits completely in $K$. The $\mathfrak{q}_{i}$ have the same meaning as in case ii).

The abelian $L$-functions we use will be defined in terms of the set $S$. Let $G=\operatorname{Gal}(K / k)$, which is abelian of cardinality $[K: k]=n$. Let $\widehat{G}=$ $\operatorname{Hom}\left(G, \mathbb{C}^{*}\right)$ with a typical character denoted by $\chi$, and the trivial character denoted by $\chi_{0}$. The $L$-functions we work with are defined by

$$
L_{S}(s, \chi)=\prod_{\mathfrak{q} \notin S}\left(1-\chi\left(\sigma_{\mathfrak{q}}\right) N \mathfrak{q}^{-s}\right)^{-1} \quad \text { for } \Re(s)>1,
$$

where $\sigma_{\mathfrak{q}}$ denotes the Frobenius automorphism associated to the prime $\mathfrak{q}$ which is uniquely defined since all of the ramified primes are contained in $S$. It is well-known [La] that these abelian $L$-functions can all be analytically continued to the whole complex plane and all of them are actually entire functions except for when $\chi=\chi_{0}$, in which case there is a simple pole at $s=1$. In particular we can expand all of these functions out as Taylor series at $s=0$ and the general philosophy is to somehow interpret the lead term arithmetically. There is a simple formula [Ta] which gives the order $r(\chi)$ of the zero of $L_{S}(s, \chi)$ at $s=0$ which is very useful.

$$
r(\chi)= \begin{cases}|S|-1 & \text { if } \chi=\chi_{0} \\ \left|\left\{\mathfrak{q} \in S: \chi\left(G_{\mathfrak{q}}\right)=1\right\}\right| & \text { if } \chi \neq \chi_{0}\end{cases}
$$

where $G_{\mathfrak{q}}$ is the decomposition group of the prime $\mathfrak{q}$ (finite or infinite). Notice by assumption (c) that $S$ contains at least $r$ primes that split completely and for all of these primes $G_{\mathfrak{q}}=\{1\}$. Combining this fact with assumption (d) we see that we always have $r(\chi) \geq r$. Thus in cases ii) and iii) all of our $L$-functions will have at least first order zeros at $s=0$. Before we can state Stark's refined conjecture we need two more pieces of notation. The number of roots of unity in $K$ is denoted by $w_{K}$. If $\mathfrak{P}$ is any prime lying above our distinguished prime $\mathfrak{p}$, then ||$_{\mathfrak{P}}$ denotes the usual [O'M, p. 65] normalized valuation on $K$ with respect to $\mathfrak{P}$.

Conjecture [St4, Ta]. There exists $\varepsilon \in K$ such that

(1) For all characters $\chi \in \widehat{G}$ we have

$$
L_{S}^{(1)}(0, \chi)=-\frac{1}{w_{K}} \sum_{\sigma \in G} \chi(\sigma) \log \left|\varepsilon^{\sigma}\right|_{\mathfrak{P}},
$$

(2) $K\left(\varepsilon^{1 / w_{K}}\right)$ is an abelian Galois extension of $k$.

Note. Tate has proved this when $|S|=2$. For $|S| \geq 3$ it is also called for that 
(3) $\varepsilon \in\left\{u \in K:|u|_{\mathfrak{P}^{\prime}}=1 \quad \forall \mathfrak{P}^{\prime} \nmid \mathfrak{p}\right\}$.

We have already stated what choice we have made for $S$ in cases ii) and iii). In case i) we can set $r=1$ by taking either of the split infinite primes to be our distinguished prime $\mathfrak{p}$. We see in this case that if $|S| \geq 3$, then all of the $L$-functions satisfy $L_{S}^{(1)}(0, \chi)=0$ and by choosing $\varepsilon=1$ the conjecture is trivially satisfied. There is only one situation of interest, namely, $S=\left\{\infty_{k}, \bar{\infty}_{k}\right\}$. This situation is already special since it implies that $K$ is contained inside the Hilbert class field of $k$ and we see by the formula for $r(\chi)$ that $L_{S}^{(1)}(0, \chi)=0$ for all $\chi \neq \chi_{0}$. Tate [Ta, p. 91] gives a nice argument for this situation.

This conjecture has been studied in great detail both numerically and theoretically by Stark [St1-St4] and has some fascinating consequences. This conjecture for case iii), where the distinguished prime is a finite prime is often referred to as the Brumer-Stark conjecture. Hayes $[\mathbf{H}]$ has given a beautiful exposition of this situation which can be reformulated [Ta] to give an intriguing generalization of Stickelberger's classic theorem on factoring Gauss sums.

For the rest of this paper we will focus on case i), so for now on $K$ denotes a totally real abelian extension of the real quadratic field $k$. As we saw above, we automatically have $L_{S}^{(1)}(0, \chi)=0$ for $\chi \neq \chi_{0}$, and since we want to glean arithmetic information from the lead term of the Taylor series expansions at $s=0$, it is natural instead to study the values $L_{S}^{(2)}(0, \chi)$. We stick to our assumptions on $S$ and take the two infinite primes of $k$ as our distinguished primes, so now $r=2$. Let $\tau$ denote an embedding of $K$ into $\mathbb{R}$ that extends the non-trivial $\mathbb{Q}$-automorphism of $k$. Note that $w_{K}=2$ since $K$ is totally real. Let $S_{K}$ consist of the set of all primes in $K$ that extend the primes in $S$. Finally, let $U=\left\{u \in K:|u|_{\mathfrak{P}}=1\right.$ for all $\left.\mathfrak{P} \notin S_{K}\right\}$ be the group of $S_{K}$-units of $K$.

Question [St5]. Are there $S_{K}$-units $\varepsilon_{1}, \varepsilon_{2} \in U$ such that all of the following conditions hold

(1) For all $\chi \in \widehat{G}$ we have

$$
\frac{1}{2 !} L_{S}^{(2)}(0, \chi)=\operatorname{det}\left[\sum_{\sigma \in G} \chi(\sigma)\left(\begin{array}{cc}
-\frac{1}{2} \log \left|\varepsilon_{1}^{\sigma}\right| & -\frac{1}{2} \log \left|\varepsilon_{1}^{\sigma \tau}\right| \\
-\frac{1}{2} \log \left|\varepsilon_{2}^{\sigma}\right| & -\frac{1}{2} \log \left|\varepsilon_{2}^{\sigma \tau}\right|
\end{array}\right)\right]
$$

(2) both $K\left(\sqrt{\varepsilon_{1}}\right)$ and $K\left(\sqrt{\varepsilon_{2}}\right)$ are abelian Galois extensions of $k$,

(3) we have $K\left(\sqrt{\varepsilon_{1}}\right)=K\left(\sqrt{\varepsilon_{2}}\right)$ in (2),

(4) the numbers $\varepsilon_{1}^{\sigma}$ generate the same principal ideal as $\sigma$ runs through $G$, and the same holds among the $\varepsilon_{2}^{\sigma}$ ? 
Note. Notice that the usual absolute value || is a normalized valuation extending the distinguished prime $\infty_{k}$, and $\left|{ }^{\tau}\right|$ is a normalized valuation extending $\bar{\infty}_{k}$.

Note. Now we are assuming that $|S| \geq 3$ in order to insure that all $L^{-}$ functions have at least 2 nd order zeros at $s=0$. If there are any ramified finite primes this is automatic, if not we have to throw in a prime $\mathfrak{q}_{1}$.

\section{Relative quadratic extensions.}

The first serious testing ground for Stark's question is the case where $[K$ : $k]=2$. We study this situation in depth here. Let $G=\operatorname{Gal}(K / k)=\{1, \sigma\}$. The problem splits naturally into two pieces that seem to require different methods: i) $|S|=3$, ii) $|S| \geq 4$. We will only consider case ii) in this paper. What makes this case more accessible is that $L_{S}^{(2)}\left(0, \chi_{0}\right)=0$. Part (1) above for $\chi_{0}$ will then follow from our work on the non-trivial character.

We have $S=\left\{\infty_{k}, \bar{\infty}_{k}, \mathfrak{q}_{1}, \cdots, \mathfrak{q}_{t}\right\}$ with $t \geq 2$. If any of the $\mathfrak{q}_{i}$ are split then $r(\chi) \geq 3$ for both $\chi \in \widehat{G}$ and the question is answered by choosing $\varepsilon_{1}=\varepsilon_{2}=1$. So we assume that the $\mathfrak{q}_{i}$ are either ramified or inert. There are four infinite primes in $S_{K}$ and $t$ finite primes $\mathfrak{Q}_{1}, \ldots, \mathfrak{Q}_{t}$ where the numbering is set up so that $\mathfrak{Q}_{i} \mid \mathfrak{q}_{i}$. Thus $\left|S_{K}\right|=|S|+2$. Our main result is the following

Theorem. If $[K: k]=2$ and $|S| \geq 4$ then Stark's question can be answered in the affirmative.

Proof. The Dedekind zeta-function $\zeta_{K}(s)$ of $K$ factors as

$$
\zeta_{K}(s)=\zeta_{k}(s) L(s, \psi)
$$

where

$$
L(s, \psi)=\prod\left(1-\psi(\mathfrak{p}) N \mathfrak{p}^{-s}\right)^{-1},
$$

the product running over all finite primes in $k$, and

$$
\psi(\mathfrak{p})= \begin{cases}1 & \text { if } \mathfrak{p} \text { splits } \\ -1 & \text { if } \mathfrak{p} \text { is inert } \\ 0 & \text { if } \mathfrak{p} \text { ramifies }\end{cases}
$$

Thus

$$
\prod_{\mathfrak{P} \notin S_{K}}\left(1-N \mathfrak{P}^{-s}\right)^{-1}=\prod_{\mathfrak{p} \notin S}\left(1-N \mathfrak{p}^{-s}\right)^{-1} \cdot \prod_{\mathfrak{p} \notin S}\left(1-\psi(\mathfrak{p}) N \mathfrak{p}^{-s}\right)^{-1} .
$$


For $\mathfrak{p} \notin S$ we have $\psi(\mathfrak{p})=\chi\left(\sigma_{\mathfrak{p}}\right)$, where $\chi$ is the non-trivial character on $G$ [Ja, p. 100]. Now the primes not in $S_{K}$ are in one-to-one correspondence with the prime ideals of the Dedekind domain of $S_{K}$-integers in $K$. The Dedekind zeta-function for the $S_{K}$-integers is precisely the left hand side of (1) and the first product on the right hand side is similarly the Dedekind zeta-function for the $S$-integers of $k$. Denoting these functions by $\zeta_{S_{K}}(s)$ and $\zeta_{S}(s)$ respectively we can rewrite (1) as

$$
\zeta_{S_{K}}(s)=\zeta_{S}(s) L_{S}(s, \chi)
$$

In regard to part (1) of Stark's question we see that $\zeta_{S}(s)=L_{S}\left(s, \chi_{0}\right)$. On the other hand we have $[\mathbf{G r}]$

$$
\lim _{s \rightarrow 0} \frac{1}{s^{2}} L_{S}(s, \chi)=\lim _{s \rightarrow 0} \frac{\frac{\zeta_{S_{K}}(s)}{s^{\mid} S_{K} \mid-1}}{\frac{\zeta_{S}(s)}{s^{|S|-1}}}=\frac{-\frac{h_{S_{K}} R_{K}}{w_{K}}}{-\frac{h_{S} R_{k}}{w_{k}}}
$$

where $h_{S_{K}}, h_{S}$ are the class numbers for the $S_{K}$-integers and $S$-integers of $K$ and $k$ respectively, and $R_{K}$ and $R_{k}$ are the regulators for the $S_{K}$-units and $S$-units respectively. Thus for the non-trivial character $\chi$ we have

$$
\frac{1}{2 !} L_{S}^{(2)}(0, \chi)=\frac{h_{S_{K}} R_{K}}{h_{S} R_{k}}
$$

and now we take a closer look at these regulators.

The Dirichlet-Chevalley-Hasse Theorem [Ja] tells us that $U$ is a direct product of the group $\mu_{K}=\{1,-1\}$ with $\left|S_{K}\right|-1=|S|+1$ infinite cyclic groups. Note that $U \cap k=U_{S}=\left\{u \in k:|u|_{\mathfrak{p}}=1 \quad\right.$ for all $\left.\mathfrak{p} \notin S\right\}$.

Let $V=\left\langle v_{1}, \ldots, v_{|S|+1}\right\rangle$ where $v_{1}, \ldots, v_{|S|+1}$ is a system of fundamental units for $U$. We will assume without loss of generality that $v_{i}>0$ for $i=$ $1, \ldots,|S|+1$. Let $V^{-}=\left\{\eta \in V: N_{K / k} \eta=1\right\}$ and let $V^{+}=\{\gamma \in V$ : $\sigma \gamma=\gamma\}$. Note that if $\sigma v_{i}>0$ we have $\sigma v_{i} / v_{i}=\eta_{i} \in V^{-}$(if $\sigma v_{i}<0$, then we consider $-\sigma v_{i} / v_{i}=\eta_{i} \in V^{-}$instead). Then $v_{i}\left(\sigma v_{i}\right)=\eta_{i} v_{i}^{2}$ so that $v_{i}^{2} \in V^{-} \cdot V^{+}$. We see that $V^{-} \cdot V^{+}$is a $\mathbb{Z}$-submodule of $V$ of finite index less than $\left[V:\left\langle v_{1}^{2}, \ldots, v_{|S|+1}^{2}\right\rangle\right]=2^{|S|+1}$. Note that $V^{+}=\left\{u \in U_{S}: u>0\right\}$ and thus is a rank $|S|-1$ submodule. The submodule $V^{-}$is necessarily free as well and since $V^{-} \cap V^{+}=\{1\}$ we have $V^{-} \cdot V^{+}=V^{-} \oplus V^{+}$which implies that $V^{-}$is a free submodule of rank 2 (see [AW, p. 173]). By the invariant factor theorem, there exists a basis $w_{1}, \ldots, w_{|S|+1}$ for $V$ and positive integers $a_{1}, a_{2}$ such that $\varepsilon_{1}=w_{1}^{a_{1}}, \varepsilon_{2}=w_{2}^{a_{2}}$ forms a basis for $V^{-}$. If $u_{1}, \ldots, u_{|S|-1}$ is a system of generators for $V^{+}$, then

$$
\left[\left\langle w_{1}, \ldots, w_{|S|+1}\right\rangle:\left\langle u_{1}, \ldots, u_{|S|-1}, \varepsilon_{1}, \varepsilon_{2}\right\rangle\right]=R / R_{K}
$$


where $R$ is the regulator for the system $\left\langle u_{1}, \ldots, u_{|S|-1}, \varepsilon_{1}, \varepsilon_{2}\right\rangle$ [Wa, p. 41]. This index can also be written

$$
R / R_{K}=\left[U: U_{S} \cdot U^{-}\right]
$$

where $U^{-}=\left\{u \in U: N_{K / k} u=1\right\}$.

We can also deduce a relationship between $R$ and $R_{k}$. By definition, the regulator $R$ is the absolute value of the following determinant

$$
\left|\begin{array}{ccccccc}
\log \left|u_{1}\right|_{\mathfrak{Q}_{1}} & \cdots & \log \left|u_{1}\right|_{\mathfrak{Q}_{t-1}} & \log \left|u_{1}\right| & \log \left|u_{1}^{\tau}\right| & \log \left|u_{1}^{\sigma}\right| & \log \left|u_{1}^{\sigma \tau}\right| \\
\vdots & \ddots & \vdots & \vdots & \vdots & \vdots & \vdots \\
\log \left|u_{|S|-1}\right|_{\mathfrak{Q}_{1}} & \cdots & \log \left|u_{|S|-1}\right|_{\mathfrak{Q}_{t-1}} & \log \left|u_{|S|-1}\right| & \log \left|u_{|S|-1}^{\tau}\right| & \log \left|u_{|S|-1}^{\sigma}\right| & \log \left|u_{|S|-1}^{\sigma \tau}\right| \\
\log \left|\varepsilon_{1}\right|_{\mathfrak{Q}_{1}} & \cdots & \log \left|\varepsilon_{1}\right|_{\mathfrak{Q}_{t-1}} & \log \left|\varepsilon_{1}\right| & \log \left|\varepsilon_{1}^{\tau}\right| & \log \left|\varepsilon_{1}^{\sigma}\right| & \log \left|\varepsilon_{1}^{\sigma \tau}\right| \\
\log \left|\varepsilon_{2}\right| \mathfrak{Q}_{1} & \cdots & \log \left|\varepsilon_{2}\right|_{\mathfrak{Q}_{t-1}} & \log \left|\varepsilon_{2}\right| & \log \left|\varepsilon_{2}^{\tau}\right| & \log \left|\varepsilon_{2}^{\sigma}\right| & \log \left|\varepsilon_{2}^{\sigma \tau}\right|
\end{array}\right| .
$$

Subtracting the fourth to the last column from the second to the last and the third to the last from the last gives us

$$
R=2^{|S|-1} R_{k} \operatorname{det}\left[\sum_{\sigma \in G} \chi(\sigma)\left(\begin{array}{cc}
-\frac{1}{2} \log \left|\varepsilon_{1}^{\sigma}\right| & -\frac{1}{2} \log \left|\varepsilon_{1}^{\sigma \tau}\right| \\
-\frac{1}{2} \log \left|\varepsilon_{2}^{\sigma}\right| & -\frac{1}{2} \log \left|\varepsilon_{2}^{\sigma \tau}\right|
\end{array}\right)\right]
$$

where a factor of $2^{|S|-3}$ comes from converting $|u|_{\mathfrak{Q}}=|u|_{\mathfrak{q}}^{2}$, for $u \in k$ and $\mathfrak{q}$ being inert or ramified, in writing down $R_{k}$. Plugging (4) and (5) into (3) we find

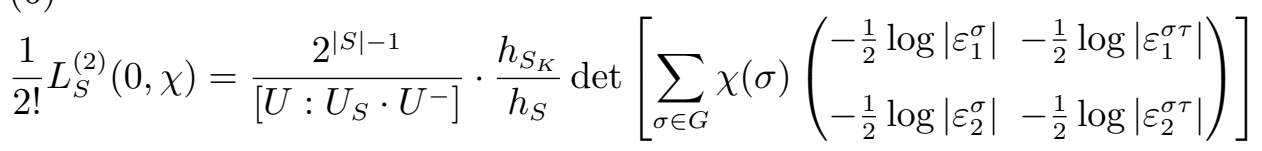

and on comparison with part (1) of Stark's question we see that we just need to analyze the indices in front of the determinant more carefully. Calling the whole product in front of the determinant $A$, we wish to show that $A \in \mathbb{Z}$. We define a homomorphism $f: U \rightarrow U$ given by $u \mapsto u / \sigma u$. By Herbrand's lemma [Iy, p. 12] we find

$$
\left[U: U_{S} \cdot U^{-}\right]=\left[f(U): f\left(U_{S} \cdot U^{-}\right)\right] \cdot\left[\operatorname{ker} f: \operatorname{ker} f \cap\left(U_{S} \cdot U^{-}\right)\right] .
$$

Notice that $\operatorname{ker} f=U_{S}$ and so the second index on the right hand side is 1 . The first index is just $\left[U^{1-\sigma}:\left\langle\varepsilon_{1}^{2}, \varepsilon_{2}^{2}\right\rangle\right]$. Furthermore,

$$
\left[U^{-}:\left\langle\varepsilon_{1}^{2}, \varepsilon_{2}^{2}\right\rangle\right]=\left[U^{-}: U^{1-\sigma}\right] \cdot\left[U^{1-\sigma}:\left\langle\varepsilon_{1}^{2}, \varepsilon_{2}^{2}\right\rangle\right]
$$


and so

$$
\frac{1}{\left[U: U_{S} \cdot U^{-}\right]}=\frac{\left|H^{1}(G, U)\right|}{\left[U^{-}:\left\langle\varepsilon_{1}^{2}, \varepsilon_{2}^{2}\right\rangle\right]}
$$

where $H^{1}(G, U)$ is the 1st cohomology group [N, p.12]. Finally, $\left[U^{-}:\left\langle\varepsilon_{1}^{2}, \varepsilon_{2}^{2}\right\rangle\right]=2^{3}$ and so we can write $A$ as

$$
A=\frac{2^{|S|-1}}{2^{3}} \cdot \frac{h_{S_{K}}}{h_{S}} \cdot\left|H^{1}(G, U)\right| .
$$

Since $|S|-1 \geq 3$ by assumption, the first index is integral. The product $\left|H^{1}(G, U)\right| h_{S_{K}} / h_{S}$ is integral also since $S$ contains all of the ramified primes [Ta, p. 105] or [CH, p. 28], and so $A$ is a positive integer.

If we consider part (1) of Stark's question for the trivial character we find

$$
\operatorname{det}\left|\begin{array}{cc}
-\frac{1}{2} \log \left|N_{K / k} \varepsilon_{1}\right| & -\frac{1}{2} \log \left|\left(N_{K / k} \varepsilon_{1}\right)^{\tau}\right| \\
-\frac{1}{2} \log \left|N_{K / k} \varepsilon_{2}\right| & -\frac{1}{2} \log \left|\left(N_{K / k} \varepsilon_{2}\right)^{\tau}\right|
\end{array}\right|=0
$$

since $N_{K / k} \varepsilon_{1}=1$. Things match up for the trivial character since we have $r\left(\chi_{0}\right)=|S|-1 \geq 3$ and so $L_{S}^{(2)}\left(0, \chi_{0}\right)=0$. Note that $\varepsilon_{1} \in U$ and so its prime factorization looks like $\left(\varepsilon_{1}\right)=\mathfrak{Q}_{1}^{b_{1}} \cdots \mathfrak{Q}_{t}^{b_{t}}$. Recalling that all of these ideals are ramified or inert we see that $\left(\sigma \varepsilon_{1}\right)$ has the same factorization. Since $\left(N_{K / k} \varepsilon_{1}\right)=(1)$, we have $b_{1}=\cdots=b_{t}=0$ and so $\varepsilon_{1}$ is a "true unit". The same goes for $\varepsilon_{2}$, so part (4) is satisfied.

With regard to part (2), there is a nice criterion that says that $K(\sqrt{\varepsilon})$ is an abelian extension of $k$ if and only if $N_{K / k} \varepsilon$ is a square in $K$ (see [T, p. 25]). We conclude that $K\left(\sqrt{\varepsilon_{1}}\right)$ and $K\left(\sqrt{\varepsilon_{2}}\right)$ are both abelian extensions of $k$. But part (3) asks that these be common abelian extensions of $k$. This can be achieved in the case where $|S| \geq 5$ since $A$ is divisible by $2^{|S|-1} / 2^{3}$ and is thus even. Multiplying the top row of the determinant in (6) by $A$ amounts to raising $\varepsilon_{1}$ to the $A$ th power. Then $\sqrt{\varepsilon_{1}^{A}} \in K$ and by adding the second row to the first row and setting $\varepsilon_{1}^{\prime}=\varepsilon_{1}^{A} \varepsilon_{2}$, we see that $\varepsilon_{1}^{\prime}$ and $\varepsilon_{2}$ satisfy all the requirements of the question. This concludes the proof of our theorem when $|S| \geq 5$. If $|S|=4$ we have $S_{K}$-units $\varepsilon_{1}, \varepsilon_{2} \in U$ satisfying parts (1) and (2) of the question, but the abelian extensions $K\left(\sqrt{\varepsilon_{1}}\right)$ and $K\left(\sqrt{\varepsilon_{2}}\right)$ of $k$ might not be same.

$$
\text { 3. }|S|=4 \text {. }
$$

We now wish to carry out a more careful analysis of the relative quadratic case when $|S|=4$ which we assume for the rest of this section. We first 
show that we can answer Stark's question affirmatively here as well except possibly for the family of situations where all of the following conditions hold:

(a) $k$ is a real quadratic field with discriminant $d_{k} \equiv 1(\bmod 8)$, i.e. 2 splits, say $(2)=\mathfrak{p}_{2} \overline{\mathfrak{p}}_{2}$,

(b) $N\left(\varepsilon_{k}\right)=1$ where $\varepsilon_{k}>1$ is the fundamental unit of $k$, so $d_{k}$ has at least two prime divisors [Co, p. 185],

(c) $K=k\left(\sqrt{\varepsilon_{k}}\right)$,

(d) the two prime ideals in $k$ above 2 ramify in $K$.

Assuming that these conditions do hold, we see that (b) and (c) insure that $K$ is totally real and that the only primes in $k$ that can ramify are $\mathfrak{p}_{2}$ and $\overline{\mathfrak{p}}_{2}$. So our conditions imply that we must choose $S=\left\{\infty_{k}, \bar{\infty}_{k}, \mathfrak{p}_{2}, \overline{\mathfrak{p}}_{2}\right\}$. Once $S$ is defined, then $A$ is uniquely defined by Equation (7). If $A$ is even, we can argue as above and finish off the question. So we can also assume

(e) $A$ is odd.

We now proceed to reduce ourselves to this recalcitrant family of situations by showing that we can answer the question affirmatively otherwise.

We already have a choice of $\varepsilon_{1}$ and $\varepsilon_{2}$ that almost works. We just have to find a way to modify them so that $K\left(\sqrt{\varepsilon_{1}}\right)=K\left(\sqrt{\varepsilon_{2}}\right)$, which is equivalent to the ratio $\varepsilon_{1} / \varepsilon_{2}$ being a square in $K$. Let $U_{K}$ be the unit group of $K$ and $M=\left\{u \in U_{K}: u>0\right\}$. By Dirichlet's unit theorem this is a free $\mathbb{Z}$-module of rank 3. Recall that $\varepsilon_{1}$ and $\varepsilon_{2}$ are in $U_{K}$ with $\varepsilon_{1}>0$ and $\varepsilon_{2}>0$. We first want to show that either the basis $\left\langle\varepsilon_{1}, \varepsilon_{2}\right\rangle$ of $V^{-}$can be extended to a basis for $M$ or that Stark's question has an affirmative answer. If $x \in V^{-}$, and $x=y^{a}$ for some $y \in M$ and $a>0$, we need to show that $y \in V^{-}$to be able to extend the basis [AW, p. 171]. Consider $N_{K / k}(y)^{a}=N_{K / k}(x)=1$ which implies $N_{K / k}(y)= \pm 1$. If the only exponents for which this happens are odd we are done since an odd $a$ implies $N_{K / k}(y)=1$. Assume $a$ is even for some $y$. Then $\varepsilon_{1}^{n_{1}} \varepsilon_{2}^{n_{2}}=y^{2 b}$ with $n_{1}, n_{2} \in \mathbb{Z}$. If $n_{1}$ and $n_{2}$ are both odd this implies $\varepsilon_{1} / \varepsilon_{2}$ is a square in $K$ and we have our common abelian extensions. If, say, $n_{1}$ is odd and $n_{2}$ is even, then $\varepsilon_{1}$ is a square in $K$. Replacing $\varepsilon_{1}$ by $\varepsilon_{1} \varepsilon_{2}$ we have the ratio $\varepsilon_{1} \varepsilon_{2} / \varepsilon_{2}$ being a square in $K$ as desired. Say $n_{1}, n_{2}$ are both even. Then $\varepsilon_{1}^{n_{1} / 2} \varepsilon_{2}^{n_{2} / 2}=y^{b}$ since both sides must be positive. Continuing in this way proves our claim.

Assuming now that $\varepsilon_{1}, \varepsilon_{2}, \varepsilon_{3}$ is a system of fundamental units for the unit group $U_{K}$ of $K$, we can write the fundamental unit $\varepsilon_{k}$ of $k$ as

$$
\varepsilon_{k}=\varepsilon_{1}^{a} \varepsilon_{2}^{b} \varepsilon_{3}^{c}
$$

where we can assume $c \geq 0$. We have $\varepsilon_{k}^{2}=N_{K / k} \varepsilon_{k}=N_{K / k}\left(\varepsilon_{1}^{a} \varepsilon_{2}^{b} \varepsilon_{3}^{c}\right)=$ $\left(N_{K / k} \varepsilon_{3}\right)^{c}$ which implies that $c=1$ or 2 . Now say $c=1$, then $\varepsilon_{1}, \varepsilon_{2}, \varepsilon_{k}$ is a 
system of fundamental units in $K$. We claim we are done in this case. The regulator for field $K$ is

$$
R(K)=\| \begin{array}{lll}
\log \left|\varepsilon_{k}\right| & \log \left|\varepsilon_{k}^{\sigma}\right| & \log \left|\varepsilon_{k}^{\sigma \tau}\right| \\
\log \left|\varepsilon_{1}\right| & \log \left|\varepsilon_{1}^{\sigma}\right| & \log \left|\varepsilon_{1}^{\sigma \tau}\right| \\
\log \left|\varepsilon_{2}\right| & \log \left|\varepsilon_{2}^{\sigma}\right| & \log \left|\varepsilon_{2}^{\sigma \tau}\right|
\end{array} \mid
$$

where the double vertical lines signify that we first want to take the determinant and then take the absolute value. Subtracting the first column from the second and adding the first column to the third gives

$$
R(K)=\| \begin{array}{ccc}
\log \left|\varepsilon_{k}\right| & 0 & 0 \\
\log \left|\varepsilon_{1}\right| & -\log \left|\varepsilon_{1}\right|+\log \left|\varepsilon_{1}^{\sigma}\right| & \log \left|\varepsilon_{1}\right|+\log \left|\varepsilon_{1}^{\sigma \tau}\right| \\
\log \left|\varepsilon_{2}\right| & -\log \left|\varepsilon_{2}\right|+\log \left|\varepsilon_{2}^{\sigma}\right| & \log \left|\varepsilon_{2}\right|+\log \left|\varepsilon_{2}^{\sigma \tau}\right| \mid
\end{array} \mid
$$

and so

$$
\frac{R(K)}{R(k)}=\| \begin{array}{ll}
-\log \left|\varepsilon_{1}\right|+\log \left|\varepsilon_{1}^{\sigma}\right| & \log \left|\varepsilon_{1}\right|+\log \left|\varepsilon_{1}^{\sigma \tau}\right| \mid \\
-\log \left|\varepsilon_{2}\right|+\log \left|\varepsilon_{2}^{\sigma}\right| & \log \left|\varepsilon_{2}\right|+\log \left|\varepsilon_{2}^{\sigma \tau}\right| \| .
\end{array} \mid
$$

Adding $1 / 2$ the first column to the second column and using the fact that $-\log \left|\varepsilon_{i}^{\tau}\right|=\log \left|\varepsilon_{i}\right|+\log \left|\varepsilon_{i}^{\sigma}\right|+\log \left|\varepsilon_{i}^{\sigma \tau}\right|$ we obtain

$$
\frac{R(K)}{R(k)}=2\left\|\sum_{\sigma \in G} \chi(\sigma)\left(\begin{array}{cc}
-\frac{1}{2} \log \left|\varepsilon_{1}^{\sigma}\right| & -\frac{1}{2} \log \left|\varepsilon_{1}^{\sigma \tau}\right| \\
-\frac{1}{2} \log \left|\varepsilon_{2}^{\sigma}\right| & -\frac{1}{2} \log \left|\varepsilon_{2}^{\sigma \tau}\right|
\end{array}\right)\right\| .
$$

By the same reasoning employed earlier we find

$$
\frac{1}{2 !} L_{S}^{(2)}(0, \chi)=\frac{h(K) R(K)}{h(k) R(k)} \cdot 2^{l}
$$

where $l$ is the number of inert finite primes in $S$, and $h(K), h(k)$ are the usual class numbers of the fields $K$ and $k$. For the situation at hand, the possible values of $l$ are 0,1 , and 2 . If $l=0$, then there is ramification in the extension $K / k$ and so $h(k) \mid h(K)[\mathbf{C H}$, p. 20]. We have

$$
\frac{1}{2 !} L_{S}^{(2)}(0, \chi)=2 \frac{h(K)}{h(k)}\left\|\sum_{\sigma \in G} \chi(\sigma)\left(\begin{array}{cc}
-\frac{1}{2} \log \left|\varepsilon_{1}^{\sigma}\right| & -\frac{1}{2} \log \left|\varepsilon_{1}^{\sigma \tau}\right| \\
-\frac{1}{2} \log \left|\varepsilon_{2}^{\sigma}\right| & -\frac{1}{2} \log \left|\varepsilon_{2}^{\sigma \tau}\right|
\end{array}\right)\right\|
$$

and with the even coefficient out front we can choose $\varepsilon_{1}$ and $\varepsilon_{2}$ in such a way that $\varepsilon_{1} / \varepsilon_{2}$ is a square in $K$. The same argument goes through for $l=1$. If 
$l=2$, the extension is unramified, but the quotient $h(K) / h(k)$ has at worst a denominator of $2\left[\mathbf{C H}\right.$, p. 21] and so $2^{3} \frac{h(K)}{h(k)}$ is still even and we are done.

Now assume that $c=2$ in Equation (8). Then we have

$$
\frac{R(K)}{R(k)}=\left\|\sum_{\sigma \in G} \chi(\sigma)\left(\begin{array}{cc}
-\frac{1}{2} \log \left|\varepsilon_{1}^{\sigma}\right| & -\frac{1}{2} \log \left|\varepsilon_{1}^{\sigma \tau}\right| \\
-\frac{1}{2} \log \left|\varepsilon_{2}^{\sigma}\right| & -\frac{1}{2} \log \left|\varepsilon_{2}^{\sigma \tau}\right|
\end{array}\right)\right\| .
$$

The cases where $l=1$ and $l=2$ can still be handled as above, but the case where $l=0$ looks like Equation (9) without the benefit of the 2 on the right hand side. Going back to Equation (8) with $c=2$ we make one further reduction. Notice that if we replace $\varepsilon_{1}$ by $\varepsilon_{1} \varepsilon_{k}$, then part (1) of Stark's question is still satisfied and $K\left(\sqrt{\varepsilon_{1} \varepsilon_{k}}\right)$ is still abelian over $k$. If $a$ is odd and $b$ is even we have $\varepsilon_{k}=\varepsilon_{1} \eta^{2}$ for some unit $\eta$, and by replacing $\varepsilon_{1}$ by $\varepsilon_{1} \varepsilon_{k} \varepsilon_{2}$ we have the ratio $\varepsilon_{1}^{2} \eta^{2} \varepsilon_{2} / \varepsilon_{2}$ being a square in $K$ as desired. The same type of argument goes through for $a$ even, $b$ odd, and $a$ odd, $b$ odd. Finally, for $a$ even, $b$ even, and $c=2$ we notice that $\sqrt{\varepsilon_{k}} \in K$ which implies that $K=$ $k\left(\sqrt{\varepsilon_{k}}\right)$. If $N\left(\varepsilon_{k}\right)=-1$, then $K$ wouldn't be totally real and so this situation can only arise when $N\left(\varepsilon_{k}\right)=1$. Looking at the field $K=k\left(\sqrt{\varepsilon_{k}}\right)=\mathbb{Q}\left(\sqrt{\varepsilon_{k}}\right)$, the conjugates over $\mathbb{Q}$ of $\sqrt{\varepsilon_{k}}$ are $\pm \sqrt{\varepsilon_{k}}, \pm 1 / \sqrt{\varepsilon_{k}}$ and all possible non-trivial automorphisms have order 2 so that $\operatorname{Gal}(K / \mathbb{Q})=\mathbb{Z}_{2} \times \mathbb{Z}_{2}$. The only possible ramification that can occur in $k\left(\sqrt{\varepsilon_{k}}\right) / k$ is at the primes in $k$ lying above 2 . Since the only case left over from above is where $l=0$, we need two prime ideals above 2 in $k$ which both ramify. We have now reduced ourselves to the family of situations satisfying (a), (b), (c), (d) and (e).

In order to find an affirmative answer for this family as well we need a few lemmas.

Lemma 1. If $d_{k} \equiv 1(\bmod 8)$ is the discriminant of a real quadratic number field $k$ with $N\left(\varepsilon_{k}\right)=1$, then $k\left(\sqrt{\varepsilon_{k}}\right)=\mathbb{Q}\left(\sqrt{d_{1}}, \sqrt{d_{2}}\right)$ where $d_{k}=d_{1} d_{2}$ and $d_{1}, d_{2}$ are both integers greater than 1 .

Proof. The fundamental unit is $\varepsilon_{k}=a+b \sqrt{d_{k}}$ where $a>1$ and $b$ are positive integers. It is easy to verify that $\sqrt{\varepsilon_{k}}=(\sqrt{2(a+1)}+\sqrt{2(a-1)}) / 2$. Let $2(a+1)=u^{2} d_{1}$ and $2(a-1)=v^{2} d_{2}$ with $d_{1}$ and $d_{2}$ square-free positive integers. We have $4\left(a^{2}-1\right)=u^{2} v^{2} d_{1} d_{2}$ or $x^{2} d_{k}=t^{2} d_{1} d_{2}$. Since $d_{k}$ is squarefree we find $w^{2} d_{k}=d_{1} d_{2}$ and $w \mid d_{1}$ and $w \mid d_{2}$ since $d_{1}$ and $d_{2}$ are square-free. This implies $w \mid u^{2} d_{1}-v^{2} d_{2}=4$ so $w=1$ or 2 . We want to show that $w \neq 2$. If $d_{1} d_{2}=4 d_{k}$, then $d_{1}=2 r$ and $d_{2}=2 s$ with $r s=d_{k}$ and either $r \equiv s \equiv 1(\bmod 4)$ or $r \equiv s \equiv 3(\bmod 4)$. This implies that $u^{2} r-v^{2} s=2$ or $u^{2}-v^{2} \equiv 2(\bmod 4)$ which has no solutions. Thus $d_{k}=d_{1} d_{2}$ and $d_{1}, d_{2}>1$ since otherwise we would have $\sqrt{\varepsilon_{k}} \in k$ which can't happen. 
Lemma 2. If $p$ is a prime congruent to 3 modulo 4 and $\varepsilon=a+b \sqrt{p}>1$ is the fundamental unit of $k_{1}=\mathbb{Q}(\sqrt{p})$, then $2 \varepsilon$ is a square in $k_{1}$.

Proof. We automatically have $N(\varepsilon)=1$ and so $\varepsilon=(1+\varepsilon) /(1+\bar{\varepsilon})=$ $(1+\varepsilon)^{2} / 2(1+a)$. Let $2(1+a)=u^{2} d_{1}$ where $d_{1}$ is assumed square-free. By the same argument as above in Lemma 1 we conclude that $d_{1}=2$ or $d_{1}=2 p$.

Comment. We can refine Lemma 2. Notice that $N(1+\varepsilon)=2(1+a)$. Working modulo 8 it is easy to see that $2 u^{2}$ is not the norm of an integer from $\mathbb{Q}(\sqrt{p})$ when $p \equiv 3(\bmod 8)$. Similarly, one finds that $2 p u^{2}$ is not the norm of an integer from $\mathbb{Q}(\sqrt{p})$ when $p \equiv 7(\bmod 8)$. So $d_{1}=2 p$ when $p \equiv 3$ $(\bmod 8)$ and $d_{1}=2$ when $p \equiv 7(\bmod 8)$.

Our approach now boils down to a consideration of several cases and an appeal to genus theory [Co, pp. 225-226]. By Lemma 1, the three quadratic subfields of $K$ will be $k_{1}=\mathbb{Q}\left(\sqrt{d_{1}}\right), k_{2}=\mathbb{Q}\left(\sqrt{d_{2}}\right)$, and and $k=\mathbb{Q}\left(\sqrt{d_{k}}\right)$. By the conductor-discriminant formula [Wa, p. 27], the discriminant of $K$ is given by $d_{K}=d_{k_{1}} d_{k_{2}} d_{k}$. The norm of the relative discriminant of the extension $K / k$ can be obtained from the formula $d_{K}=\left(N D_{K / k}\right)\left(d_{k}\right)^{2}$ [Lo, p. 82].

Case $1 . d_{k}$ is the product of two primes $p_{1}, p_{2}$ where either $p_{1} \equiv p_{2} \equiv 1$ $(\bmod 4)$ or $p_{1} \equiv p_{2} \equiv 3(\bmod 4)$. In either case we have $K=\mathbb{Q}\left(\sqrt{p_{1}}, \sqrt{p_{2}}\right)$. If $p_{1} \equiv p_{2} \equiv 1(\bmod 4)$ we find $N D_{K / k}=1$ which implies there is no ramification and so these fields do not belong to our family. On the other hand, if $p_{1} \equiv p_{2} \equiv 3(\bmod 4)$ we find $N D_{K / k}=16$ and so both primes above 2 in $k$ ramify. As Kubota showed (see in particular [K, p. 74]), a fundamental system of units for $K$ in this special case is $\varepsilon_{1}=\gamma_{1}, \varepsilon_{2}=$ $\sqrt{\gamma_{1} \gamma_{2}}$, and $\varepsilon_{3}=\sqrt{\varepsilon_{k}}$ where $\gamma_{1}>1, \gamma_{2}>1$ are the fundamental units for $k_{1}$ and $k_{2}$. The class number of $K$ in this particular case is given by $h(K)=h\left(k_{1}\right) h\left(k_{2}\right) h(k)[\mathbf{K}$, p. 80] and so

$$
\frac{1}{2 !} L_{S}^{(2)}(0, \chi)=h\left(k_{1}\right) h\left(k_{2}\right)\left\|\sum_{\sigma \in G} \chi(\sigma)\left(\begin{array}{cc}
-\frac{1}{2} \log \left|\varepsilon_{1}^{\sigma}\right| & -\frac{1}{2} \log \left|\varepsilon_{1}^{\sigma \tau}\right| \\
-\frac{1}{2} \log \left|\varepsilon_{2}^{\sigma}\right| & -\frac{1}{2} \log \left|\varepsilon_{2}^{\sigma \tau}\right|
\end{array}\right)\right\| .
$$

We now verify that the two $S_{K}$-units $\eta_{1}=2 \varepsilon_{1} \varepsilon_{2}$ and $\eta_{2}=\varepsilon_{2}^{h\left(k_{1}\right) h\left(k_{2}\right)}$ satisfy Stark's question. Clearly $N_{K / k} \varepsilon_{1}=1$. To see that $N_{K / k} \varepsilon_{2}=1$, let $\sqrt{\gamma_{1}}=$ 


$$
\begin{gathered}
\left(u_{1} \sqrt{2}+v_{1} \sqrt{2 p_{1}}\right) / 2 \text { and } \sqrt{\gamma_{2}}=\left(u_{2} \sqrt{2}+v_{2} \sqrt{2 p_{2}}\right) / 2 . \text { Then } \\
N_{K / k} \varepsilon_{2}=\left(\frac{u_{1} \sqrt{2}+v_{1} \sqrt{2 p_{1}}}{2}\right)\left(\frac{u_{1} \sqrt{2}-v_{1} \sqrt{2 p_{1}}}{2}\right) \\
\cdot\left(\frac{u_{2} \sqrt{2}+v_{2} \sqrt{2 p_{2}}}{2}\right)\left(\frac{u_{2} \sqrt{2}-v_{2} \sqrt{2 p_{2}}}{2}\right) \\
=1
\end{gathered}
$$

by the comment above. The $L$-function values match up and $\eta_{1} / \eta_{2}$ is a square in $K$ by Lemma 2 and the fact that $h\left(k_{1}\right) h\left(k_{2}\right)$ is odd by genus theory.

Case 2. $d_{k}$ is the product of three primes $p_{1}, p_{2}$ and $q$ where $p_{1} \equiv p_{2} \equiv 3$ $(\bmod 4)$ and $q \equiv 1(\bmod 4)$ (the situation where all three are congruent to 1 modulo 4 is eliminated since $\left.N D_{K / k}=1\right)$. The three quadratic subfields look like $k_{1}=\mathbb{Q}\left(\sqrt{p_{1}}\right), k_{2}=\mathbb{Q}\left(\sqrt{p_{2} q}\right)$, and $k$ by Lemma 1 (we can eliminate the situation where $k_{1}=\mathbb{Q}(\sqrt{q})$ and $k_{2}=\mathbb{Q}\left(\sqrt{p_{1} p_{2}}\right)$ since $\left.N D_{K / k}=1\right)$. If $q \equiv 5(\bmod 8)$, then we find using Hilfssatz 10 in $[\mathbf{K}]$ that $\varepsilon_{1}=\gamma_{1}, \varepsilon_{2}=$ $\gamma_{2}$, and $\varepsilon_{3}=\sqrt{\varepsilon_{k}}$ are a system of fundamental units for $K$. Now we have $h(K) / h(k)=h\left(k_{1}\right) h\left(k_{2}\right) / 2$ and we know by genus theory that $2 \mid h\left(k_{2}\right)$. The most interesting situation would be if $2 \| h\left(k_{2}\right)$. Then we would use $\eta_{1}=2 \varepsilon_{1} \varepsilon_{2}$ and $\eta_{2}=\varepsilon_{2}^{h\left(k_{1}\right) h\left(k_{2}\right) / 2}$. If $q \equiv 1(\bmod 8)$, we find a fundamental system of units that looks like the $q \equiv 5(\bmod 8)$ situation above (type ii) in Satz 1 of $[\mathbf{K}]$ ) or like $\varepsilon_{1}=\gamma_{1}, \varepsilon_{2}=\sqrt{\gamma_{1} \gamma_{2}}$, and $\varepsilon_{3}=\sqrt{\varepsilon_{k}}$ (type v) in Satz 1). The latter set up is even easier since $h(K) / h(k)=h\left(k_{1}\right) h\left(k_{2}\right)$ is now even and we can choose $\eta_{1}=\varepsilon_{1}, \eta_{2}=\varepsilon_{1} \varepsilon_{2}^{h\left(k_{1}\right) h\left(k_{2}\right)}$.

Case 3. We now assume that $d_{k}$ is the product of 4 or more primes. We use Lemma 1 to describe the various possibilities we can have for the three quadratic subfields. We can eliminate a lot of possibilities by calculating $N D_{K / k}$ and finding it equal to 1 . For the remaining possibilities, an appeal to genus theory supplies us with enough powers of 2 to always enable us to find an affirmative answer.

Notice that all solutions $\varepsilon_{1}, \varepsilon_{2} \in U$ we found above satisfy part (4) of the question. The most interesting parts of Stark's question are (1) and (2) because of the obvious analogy with his conjecture. Parts (3) and (4) are more provisional and might need to be modified or dropped based on further study.

Acknowledgements. This paper gives a partial summary of work completed during the author's Ph.D. studies [T]. It is a pleasure to acknowledge 
the following people who were extremely helpful: David Dummit, Karl Rubin, and Jonathan Sands. I would especially like to thank my thesis advisor Harold Stark for all of his help and encouragement.

\section{References}

[AW] W. Adkins and S. Weintraub, Algebra, Springer-Verlag, New York, Berlin, 1992.

[Co] H. Cohn, Advanced number theory, Dover, New York, 1980.

$[\mathrm{CH}]$ P. Conner and J. Hurrelbrink, Class number parity, World Scientific, Singapore, 1988.

[Gr] B.H. Gross, On the values of abelian L-functions at $s=0$, J. Fac. Sci. Univ. Tokyo, 35 (1988), 177-197.

[H] D.R. Hayes, Brumer elements over a real quadratic base field, Expositiones Mathematicae, 8 (1990), 137-184.

[Iy] S. Iyanaga, Class-field theory notes, Chicago, 1961.

[Ja] G. Janusz, Algebraic number fields, Academic Press, New York, 1973.

[K] T. Kubota, Über den bizyklischen biquadratischen zahlkörper, Nagoya Math. J., 10 (1955), 65-85.

[La] S. Lang, Algebraic number theory, Springer-Verlag, New York, Berlin, 1986.

[Lo] R. Long, Algebraic number theory, Marcel Dekker, Inc., New York, Basel, 1977.

[N] J. Neukirch, Class field theory, Springer-Verlag, New York, Berlin, 1986.

[O'M] O.T. O'Meara, Introduction to quadratic forms, Springer-Verlag, New York, Berlin, 1963.

[St1] H.M. Stark, Class fields for real quadratic fields and L-series at 1 , Algebraic number fields (A. Fröhlich, ed.), Academic Press, London, (1977), 355-375.

[St2] Hilbert's twelfth problem and L-series, Bulletin of the AMS, 83 (1977), 1072-1074.

[St3] , L-functions at $s=1$. III, Adv. in Math., 22 (1976), 64-84.

[St4] L L-functions at $s=1$ IV, Adv. in Math., 35 (1980), 197-235.

[St5] - Derivatives of L-series at $s=0$, Abstracts of the AMS, 1 (1980), 28.

[T] B.A. Tangedal, The conjectures of Sands and Stark for special values of abelian L-functions, Ph.D. Thesis, U.C.S.D., San Diego, 1994.

[Ta] J. Tate, Les conjectures de Stark sur les fonctions $L$ d'Artin en $s=0$, Birkhäuser, Boston, 1984.

[Wa] L. Washington, Introduction to cyclotomic fields, Springer-Verlag, New York, Berlin, 1982.

Received August 30, 1995.

College of Charleston

Charleston, SC 29424-0001

E-mail address: tangedal@math.cofc.edu 